\title{
氨基-唑基双功能化离子液体相变吸收剂捕集 $\mathrm{CO}_{2}$ 机制
}

\author{
吕碧洪, 詹晓慧, 李昕, 周作明, 荆国华 ${ }^{*}$ \\ 华侨大学化工学院, 厦门 361021 \\ *通讯作者, E-mail: zhoujing@hqu.edu.cn \\ 收稿日期: 2021-01-31; 接受日期: 2021-04-02; 网络版发表日期: 2021-10-08 \\ 华侨大学中青年教师优秀科技创新人才计划(编号: ZQN-YX603)和国家自然科学基金(编号: 21808074)资助项目
}

摘要相变吸收剂是一类极具节能潜力的 $\mathrm{CO}_{2}$ 吸收剂, 但现有胺类相变吸收剂存在富相溶液黏度大、再生效 率低的缺陷. 本文设计合成了一种二乙烯三胺咪唑功能化离子液体, 将其溶于乙醇-水混合溶剂中, 创新性地构建 了氨基-唑基双功能离子液体-乙醇-水相变吸收剂用于 $\mathrm{CO}_{2}$ 捕集. [DETAH] $[\mathrm{Im}]-$ 乙醇- $\mathrm{H}_{2} \mathrm{O}$ 相变吸收剂吸收 $\mathrm{CO}_{2}$ 前为 均相溶液, 而吸收饱和后发生液液相变, $\mathrm{CO}_{2}$ 产物主要富集于下层水相, 当乙醇在混合溶剂中的比例为 $70 v / v \%$ 时, 富相溶液吸收容量占总 $\mathrm{CO}_{2}$ 容量 $(1.548 \mathrm{~mol} \mathrm{CO} / \mathrm{mol}$ absorbent) $92 \%$ 而体积仅占 $32 \%$; 且在乙醇作为再生活化剂时, 富相溶液经过4次再生仍保持其初始负载的 $90 \%$ 以上. [DETAH] [Im] 中的氨基和唑基官能团与 $\mathrm{CO}_{2}$ 均可发生化学 反应，保证了体系高效的 $\mathrm{CO}_{2}$ 容量. 且 $[\mathrm{Im}]-\mathrm{H}$ 和乙醇均可增强 $\mathrm{CO}_{2}$ 反应产物氨基甲酸酯分解转化，形成易分解的 $\mathrm{HCO}_{3}{ }^{-} / \mathrm{CO}_{3}{ }^{2-}$ 和碳酸乙酯, 确保富相溶液的高再生效率. 由于密度大的产物在水中的氢键作用和极性均强于乙醇, 促使产物向下层水溶液中富集, 而密度小的乙醇处于上层, 最终形成液-液相变.

关键词二氧化碳, 功能化离子液体, 相变吸收剂, 机理

\section{1 引言}

2016年11月《巴黎协定》生效后，共同应对气候 变化已成为全球共识. 2020 年, 习近平总书记在七十 五届联合国大会一般性辩论上的讲话指出, 我国二氧 化碳 $\left(\mathrm{CO}_{2}\right)$ 排放力争于 2030 年前达到峰值, 努力争取 2060 年前实现碳中和 ${ }^{[1,2]}$. 在此愿景下, 碳捕集利用与 封存技术 $(\mathrm{CCS})$ 是实现我国乃至全球碳中和目标的重 要途径之一 ${ }^{[3,4]}$. 目前, 胺类吸收剂, 尤其是单乙醇 (MEA)的化学吸收已在工业中运用, 但存在易发泡、
易氧化降解、再生能耗高等缺陷. 以MEA为例, 由于 溶剂水具有较大的比热容和蒸发焓, 再生过程中大量 能耗主要用于水分加热和蒸发过程，因该过程消耗的 热量占总能耗的 $70 \% \sim 80 \%{ }^{[5 \sim 7]}$, 限制了其大规模的工 业应用 ${ }^{[8]}$. 如何降低碳捕集技术的成本, 尤其是再生能 耗成为该领域研究的一大重点.

相变吸收剂在这样的情形下应运而生. 该类新型 吸收剂在室温下为均相, 通过温度或容量调节, 将变 成液-液或者液-固两相, 且 $\mathrm{CO}_{2}$ 仅富集于其中一相 ${ }^{[9,10]}$; 因而只需将 $\mathrm{CO}_{2}$ 富相溶液送至再生塔内解吸, 以大幅

引用格式: Lv B, Zhan X, Li X, Zhou Z, Jing G. Mechanism of $\mathrm{CO}_{2}$ capture into amino-azolyl dual-functionalized ionic liquid biphasic solvent. Sci Sin Chim, 2021, 51: 1660-1670, doi: 10.1360/SSC-2021-0026 
度减少进入再生塔内的液体流量，进而减少该体系的 再生能耗. 目前已有的相变吸收剂大多是胺类试 剂 $^{[11,12]}$. Wang 等 ${ }^{[13]}$ 开发了单乙醇胺/正丙醇相变吸收 剂, 其再生能量降低了 $39.85 \%$, 循环容量从 $1.01 \mathrm{~mol}$ $\mathrm{CO}_{2} / \mathrm{kg}$ absorbent增加到 $2.51 \mathrm{~mol} / \mathrm{kg}$. Z Z 等 $^{[14]}$ 开发的 $\mathrm{MEA}+\mathrm{SA}+\mathrm{H}_{2} \mathrm{O}$ 吸收剂, 与 $30 \mathrm{wt} \%$ 单乙醇胺相比, 其再 生时所需要的能量减少了 $43.6 \%$. Shen等 ${ }^{[15]}$ 将脯氨酸 钾与乙醇混合形成分相吸收剂, 其吸收容量为 $0.55 \mathrm{~mol} \mathrm{CO}_{2} / \mathrm{kg}$ absorbent, 在 $120^{\circ} \mathrm{C}$ 加热且 $0.2 \mathrm{~L} / \mathrm{min}$ $\mathrm{N}_{2}$ 吹扫下再生效率高达 $90.9 \%$. 虽然, 相变吸收剂能有 效降低捕集 $\mathrm{CO}_{2}$ 的能耗, 但已报道胺类相变吸收剂存 在富相溶液黏度大的缺陷 ${ }^{[16]}$, 这将加剧设备腐蚀并导 致传输成本增加; 且一般是在 $\mathrm{N}_{2}$ 吹扫加热条件才具有 较高的再生效率, 最终收集到的气体为 $\mathrm{N}_{2} / \mathrm{CO}_{2}$ 混合气 体, 不利于 $\mathrm{CCS}$ 后期的封存处理, 该技术要求 $\mathrm{CO}_{2}$ 封存 浓度大于 $95 \%$.

离子液体(ionic liquids, ILs)作为一种绿色溶剂, 因 具有性质稳定、结构可设计等优点 ${ }^{[17,18]}$, 通过调整阴 阳离子组合或引入特定功能化基团, 可获得具有特殊 功能、任务专一的功能化离子液体 ${ }^{[19]}$. 近期, 有研究 发现, 一些离子液体、功能化离子液体通过调控也具 有相变特性 ${ }^{[20]}$. Seo 等 ${ }^{[21]}$ 笁选了一系列相变离子液体 (PCILS)用于 $\mathrm{CO}_{2}$ 吸收, 发现它们在室温下呈固体, 而 与 $\mathrm{CO}_{2}$ 反应后能够变成液体, 因而可利用融化热提供 部分 $\mathrm{CO}_{2}$ 解吸的热量, 以降低 $\mathrm{CO}_{2}$ 再生能耗. 然而, 他 们研发的 $\left[\mathrm{P}_{2222}\right][\mathrm{BnIm}]$ 富相溶液在 $20^{\circ} \mathrm{C}$ 时黏度高达 $10000 \mathrm{mPa} \mathrm{s}^{[22]}$. Kassim等 ${ }^{[23]}$ 则将MEA和离子液体 $[\mathrm{BMIM}]\left[\mathrm{NTf}_{2}\right]$ 溶于环丁砜中组成相变吸收剂, 发现 $30 \mathrm{wt} \% \mathrm{MEA}+70 \mathrm{wt} \%$ 环丁砜溶液的分相效果最好, 但 其 5 次再生效率仅为 $46 \%$. 此外, 氨基功能化离子液体 ([TETA]Br)也被证实与醇胺、水可形成三元相变吸收 剂, 且[TETA]Br中 $[\mathrm{TETA}]$ 与 $\mathrm{CO}_{2}$ 发生摩尔比为 $2: 1$ 的吸 收反应, 吸收饱和后的 $\mathrm{CO}_{2}$ 容量为 $2.631 \mathrm{~mol} \mathrm{CO}_{2} / \mathrm{L} \mathrm{ab}-$ sorbent ${ }^{[24]}$. 因此, 基于功能化离子液体的新型相变吸收 剂, 兼具功能化离子液体及相变吸收剂的优点, 且弥补 各自的缺陷, 可为碳捕集提供高效、经济的新思路.

本文设计合成了一种氨基-唑基双功能化离子液 体([DETAH] [Im]), 其由二乙烯三胺阳离子 $\left([\mathrm{DETAH}]^{+}\right)$ 和非质子杂环阴离子 ([Im $\left.]^{-}\right)$构成. 因活性官能团 $\left([\mathrm{Im}]^{-}\right)$不仅与 $\mathrm{CO}_{2}$ 发生等摩尔反应还可促进氨基甲酸 酯水解成易分解的物质 $\left(\mathrm{HCO}_{3}{ }^{-} / \mathrm{CO}_{3}{ }^{2-}\right)$, 该离子液体具
有良好的 $\mathrm{CO}_{2}$ 捕集和再生性能 ${ }^{[25]}$. 通过醇-水混合溶剂 的相变调控, 创新性地构建了功能化离子液体-醇-水 三元相变体系. 其中, [DETAH][Im]作为主吸收剂, 保 证体系良好的热稳定和 $\mathrm{CO}_{2}$ 捕集性能; 乙醇作为分相 试剂, 促使 $\mathrm{CO}_{2}$ 吸收产物在混合溶剂中分相; 而水作为 溶剂, 可确保富相溶液的低黏度. 本文考察了 [DETAH] $[\mathrm{Im}]$-乙醇- $\mathrm{H}_{2} \mathrm{O}$ 新型相变吸收剂捕集 $\mathrm{CO}_{2}$ 吸收-解吸性 能, 并通过 ${ }^{13} \mathrm{C} N M R$ 和量子化学计算的分析手段, 阐明 了该相变吸收剂捕集 $\mathrm{CO}_{2}$ 的反应机理和相变机制.

\section{2 实验部分}

\section{1 试剂与仪器}

二乙烯三胺(DETA， $\geq 99.90 \%$, 成都西亚化学试剂 有限公司, 中国)、乙醇 $(\mathrm{EtOH}, \geq 99.90 \%$, 上海阿拉丁 有限公司, 中国)、咪唑([Im]-H, $\geq 99.90 \%$, 国药集团化 学试剂有限公司, 中国)、氛代水 $\left(\mathrm{D}_{2} \mathrm{O}, \geq 99.90 \% \mathrm{D}\right.$, Sigma-Aldrich Chemistry, 美国)、二氧化碳和氮气 $\left(\mathrm{CO}_{2} 、 \mathrm{~N}_{2}, \geq 99.99 \mathrm{vol} \%\right.$, 厦门空分特气体有限公司, 中国), 实验所用水均为超纯水.

核磁共振碳谱仪 $\left({ }^{13} \mathrm{C}\right.$ NMR, AVANCE III $500 \mathrm{MHz}$, 瑞士); pH计(FE20, Mettler Toledo, 瑞士).

\section{2 氨基-唑基双功能化离子液体制备}

氨基-㛗基双功能化离子液体([DETAH][Im])采用 酸碱中和一步合成法制备 ${ }^{[7]}$. 分别称取等摩尔的DETA 和Im溶于乙醇-水混合液 $(4: 1, v / v \%)$ 中, 在 $25^{\circ} \mathrm{C}$ 条件下 磁力搅拌 $24 \mathrm{~h}$, 使酸碱充分发生中和反应. 随后将混合 液转移至圆底烧瓶中, 在 $60^{\circ} \mathrm{C}$ 条件下置于旋转蒸发仪 中去除溶剂后所得产物即为[DETAH][Im]. 根据实验 设计, 配置一定体积比的乙醇-水溶剂, 溶解适量的 [DETAH] [Im]后即得特定浓度下的[DETAH][Im]-乙 醇- $\mathrm{H}_{2} \mathrm{O}$ 相变吸收剂.

\section{3 实验方法}

配制 $1 \mathrm{kmol} / \mathrm{m}^{3}$ 的 $\left[\right.$ DETAH][Im]-乙醇- $\mathrm{H}_{2} \mathrm{O}$ 相变吸 收剂, 借助鼓泡吸收法在 $313.15 \mathrm{~K}$ 、常压下吸收 $\mathrm{CO}_{2}$, 吸收饱和后相变吸收剂由均相变为液液两相, 将下层 富相溶液分离后置于 $393.15 \mathrm{~K}$ 、常压油浴锅中再生. 吸收-解吸过程实验装置详见图S1 (网络版补充材料). 在吸收-解吸过程中分别对不同阶段产物取样, 以 $\mathrm{D}_{2} \mathrm{O}$ 
为溶剂, 进行 ${ }^{13} \mathrm{C} N \mathrm{NMR}$ 测试, 分析产物组分在反应过程 中的变化及不同相层间的分布情况. 样品中的 $\mathrm{CO}_{2}$ 容 量采用酸解方法测量, 实验装置和测量方法详见图 S2.

吸收剂溶液的吸收速率 $\left(r_{\mathrm{a}}, \mathrm{mol} \mathrm{CO}_{2} /(\mathrm{mol}\right.$ absorbent $\min )$ ) 可以通过测量㿝膜流量计进、出口气体流 速的差值得出. 吸收剂的吸收容量 $\left(L_{\mathrm{a}}, \mathrm{mol} \mathrm{CO}_{2} / \mathrm{mol}\right.$ absorbent) 可通过 $r_{\mathrm{a}}$ 对时间 $(t, \mathrm{~min})$ 积分得到. 再生效率 $(\eta, \%)$ 为第 $n$ 次吸收 $\mathrm{CO}_{2}$ 所测得的容量和初次测得的吸 收容量的比值. 具体计算公式见网络版补充材料.

\section{4 量子化学计算}

除了核磁表征外，吸收剂捕集 $\mathrm{CO}_{2}$ 的反应及相变 机理结合密度泛函理论(DFT)方法, 采用高斯09软件 (B3LYP/6-31++ $\mathrm{G}^{* *}$ 基组)和Material Studio 7.0软件 (DMOL3 模块)进行量子化学模拟 ${ }^{[26-28]}$. 所需计算公式 如下 ${ }^{[29 \sim 32]}$ :

$\Delta E_{\text {LUMO-Hомо }}=E_{\text {LUMO }}-E_{\text {HОMO }}$

$E_{\mathrm{a}}=E_{\text {transition state }}-E_{\text {reactant }}$

其中 $E_{\text {LUMO }}(\mathrm{eV})$ 和 $E_{\text {HOMO }}(\mathrm{eV})$ 分别是最低的未占据 分子轨道的能量和最高的未占据分子轨道的能量; $\Delta E_{\text {LUмо-номо }}(\mathrm{eV})$ 为 $E_{\mathrm{LUMO}}$ 和 $E_{\mathrm{HOMO}}$ 间的能量差值; 所 有模拟计算数据都通过叠加误差(BSSE)校正和零点 能量(ZPE)校正获得保证数据的准确性.

\section{3 结果与讨论}

\section{1 [DETAH] [Im]-乙醇- $\mathrm{H}_{2} \mathrm{O}$ 相变吸收剂的构建}

相变吸收剂的性能与其组成息息相关, 因此, 在保
持 [DETAH][Im] 的总摩尔浓度为 $1 \mathrm{kmol} / \mathrm{m}^{3}$ 的情况下， 首先对 [DETAH][Im]-乙醇- $\mathrm{H}_{2} \mathrm{O}$ 三元体系的组分配比 进行优化. 如表1所示, 在主吸收剂浓度一定的情况下, 乙醇与水的体积配比决定了三元吸收剂捕集 $\mathrm{CO}_{2}$ 的容 量及相变特性. 当乙醇比例低于 $50 v / v \%$ 时，该体系吸 收 $\mathrm{CO}_{2}$ 前后均保持均相, 且其 $\mathrm{CO}_{2}$ 容量随着体积分数的 增大而增大. 当乙醇比例大于 $60 v / v \%$ 后，该均相体系 饱和溶液发生液-液相变, $\mathrm{CO}_{2}$ 主要富集在溶液下层. 乙 醇比例从 $60 v / v \%$ 增加至 $80 v / v \%$, 体系的容量基本保持 在 $1.5 \mathrm{~mol} \mathrm{CO} / \mathrm{mol}$ absorbent左右; 但富相溶液的容量 逐渐增大，其体积则从 $36 \%$ 降至 $28 \%$ ，且都有 $90 \%$ 以上 的 $\mathrm{CO}_{2}$ 富集于富相溶液中. 实验结果说明, 通过改变乙 醇在混合溶剂中的比例, 可以调节 $\mathrm{CO}_{2}$ 在本三元体系 中的溶解情况, 进而调控相变行为.

[DETAH] [Im]-乙醇- $\mathrm{H}_{2} \mathrm{O}$ 相变吸收剂捕集 $\mathrm{CO}_{2}$ 过程 的吸收速率及容量变化如图1所示. 随着反应时间的增 加, 吸收速率逐渐减小而容量逐渐增大; 与表 1 结果类 似, 在60 80 v/v\%范围中, 溶剂中乙醇的比例对相变体 系的性能影响不大，速率与容量在相同反应时间内数 值相近. 基于表 1 的结果, 以及相变吸收剂富相溶液容 量宜超过总容量 $90 \%$ 且体积占总体积的 $30 \%$ $40 \%$ 的 条件, 选取 $70 v / v \%$ 乙醇配比作为最佳比例进行后续的 实验研究. 此时, 相变体系饱和后下层富相溶液的容 量、体积分别占总体积的 $92.1 \%$ 和 $32 \%$, 且吸收饱和后 其黏度为 $9.57 \mathrm{mPa} \mathrm{s}$.

\section{2 [DETAH] $[\mathrm{Im}]-$ 一醇- $\mathrm{H}_{2} \mathrm{O}$ 相变吸收剂解吸性能}

基于前文优化，该相变吸收剂中[DETAH][Im] 的 摩尔浓度为 $1 \mathrm{kmol} / \mathrm{m}^{3}$, 乙醇配比为 $70 \mathrm{v} / \mathrm{v} \%$. 解吸条件

表 1 不同乙醇配比下相变吸收剂饱和溶液特性的对比 ${ }^{\mathrm{a})}$

Table 1 Comparison of the $\mathrm{CO}_{2}$-saturated biphasic solvent at different volume fraction of ethanol ${ }^{\mathrm{a}}$

\begin{tabular}{cccccc}
\hline \multirow{2}{*}{ EtOH配比 $(v / v \%)$} & 相变吸收剂 & 富相体积 $(\%)$ & \multicolumn{3}{c}{$\mathrm{CO}_{2}$ 负荷 $\left(\mathrm{mol} \mathrm{CO}_{2} / \mathrm{mol}_{\text {absorbent })}\right.$} \\
\cline { 4 - 6 } 20 & 否 & - & 总负荷 & 上层 \\
30 & 否 & - & 1.659 & - & - \\
40 & 否 & - & 1.684 & - & - \\
50 & 否 & - & 1.776 & - & 1.297 \\
60 & 是 & 36 & 1.850 & 0.219 & 1.425 \\
70 & 是 & 32 & 1.516 & 0.123 & 1.480 \\
\hline
\end{tabular}

a) $C_{\text {[DETAH][Im] }}: 1 \mathrm{kmol} / \mathrm{m}^{3} ; Q_{\mathrm{CO}_{2}}: 60 \mathrm{~mL} / \mathrm{min} ; V_{\mathrm{L}}: 25 \mathrm{~mL}$ 


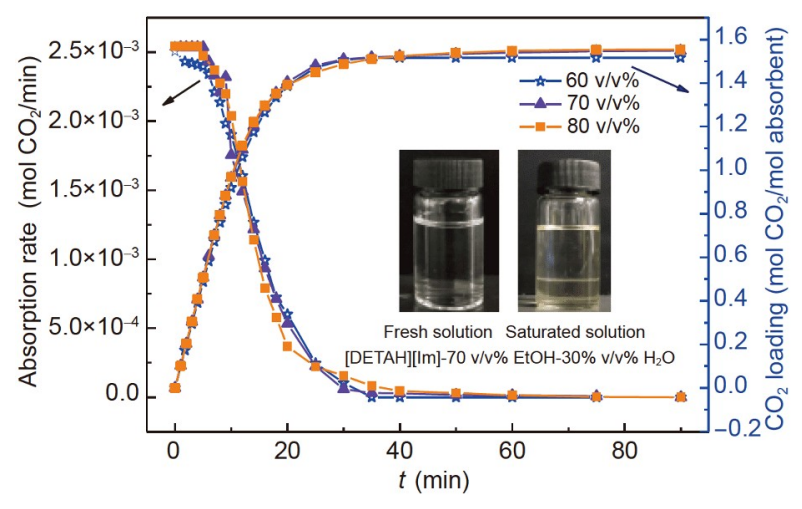

图 1 乙醇配比对 [DETAH][Im]-乙醇- $\mathrm{H}_{2} \mathrm{O}$ 相变吸收剂捕集 $\mathrm{CO}_{2}$ 的影响 $\left(C_{[\text {DETAH][Im] }}: 1 \mathrm{kmol} / \mathrm{m}^{3} ; Q_{\mathrm{CO}_{2}}: 60 \mathrm{~mL} / \mathrm{min} ; V_{\mathrm{L}}\right.$ : $25 \mathrm{~mL}$ ) (网络版彩图)

Figure 1 Effect of ethanol volume fraction on $\mathrm{CO}_{2}$ capure performance into [DETAH] $[\mathrm{Im}]-\mathrm{EtOH}-\mathrm{H}_{2} \mathrm{O}$ biphasic solution $\left(C_{\text {[DETAH][Im] }}: 1\right.$ $\mathrm{kmol} / \mathrm{m}^{3} ; Q_{\mathrm{CO}_{2}}: 60 \mathrm{~mL} / \mathrm{min} ; V_{\mathrm{L}}: 25 \mathrm{~mL}$ ) (color online)

优化时(图S3), 富相溶液单独解吸后再生效率仅为 $78.29 \%$ ，而加入少量上层贫相溶液则可适当提高富相 溶液的再生效率, 且当上层贫相溶液添加量为 $2 \mathrm{~mL}$ 时, 富相溶液再生效率提高至92.21\%。该操作虽然增加了 再生富相溶液的体积，但进入再生装置的液体体积仅 由原本的 $32 \%$ 增加至总吸收剂的 $40 \%$ ，仍然极具节能 潜力.

随后, 将再生后溶液与贫相溶液混合, 形成均相溶 液进行循环吸收-再生实验, 结果如图2所示. 随着循环 次数的增加, 饱和富相溶液的再生效率没有显著变化, 第四次再生后，[DETAH][Im]-乙醇- $\mathrm{H}_{2} \mathrm{O}$ 相变吸收剂仍 保持 $90 \%$ 的初始吸收量. 实验结果表明该相变吸收剂 具有良好的稳定性和重复利用性，与常规有机胺吸收 剂需辅助手段来保持高再生性能相比具有更好的应用 前景.

\section{3 [DETAH][Im]-乙醇- $\mathrm{H}_{2} \mathrm{O}$ 相变吸收剂捕集 $\mathrm{CO}_{2}$ 反应及相变机制}

\subsection{1 吸收-解吸反应机理}

利用 ${ }^{13} \mathrm{C}$ NMR图谱分析[DETAH][Im]-乙醇- $\mathrm{H}_{2} \mathrm{O}$ 相 变吸收剂捕集 $\mathrm{CO}_{2}$ 过程的组分变化情况，样品中可能 存在的物质结构和C原子标识如图3d所示. 图3a展示 的是吸收过程中均相溶液、饱和后上层贫相溶液与下 层富相溶液的图谱. 均相溶液在化学位移为 50.92 和 $39.97 \mathrm{ppm}$ 的位置上出现了 $[\mathrm{DETAH}]^{+}$上的两个C原子

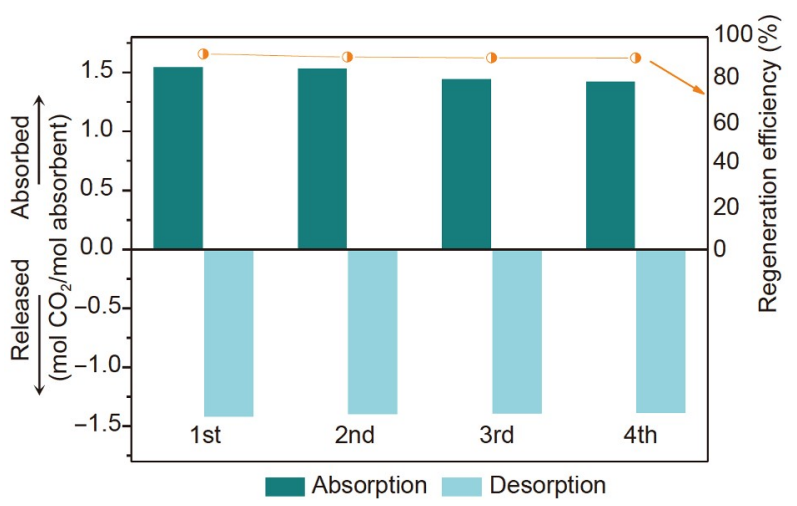

图 2 [DETAH] [ Im]-乙醇 $-\mathrm{H}_{2} \mathrm{O}$ 相变吸收剂再生特性 $\left(C_{\text {[DETAH] }[\mathrm{Im}]}: 1 \mathrm{kmol} / \mathrm{m}^{3} ; \psi_{\mathrm{EtOH}}: 70 v / v \% ; Q_{\mathrm{CO}_{2}}: 60 \mathrm{~mL} / \mathrm{min} ; V_{\mathrm{L}}\right.$ : $25 \mathrm{~mL}$; $T_{\text {absorption }}: 313.15 \mathrm{~K}$; $T_{\text {desorption }}: 393.15 \mathrm{~K}$; $t_{\text {desorption }}$ : $30 \mathrm{~min}$; $P_{\text {regeneration }}: 101.325 \mathrm{kPa}$ ) (网络版彩图)

Figure 2 Regeneration performance of [DETAH][Im]-EtOH- $\mathrm{H}_{2} \mathrm{O}$ biphasic solvent. $\left(C_{\text {[DETAH] }[\mathrm{mm}]}: 1 \mathrm{kmol} / \mathrm{m}^{3} ; \psi_{\mathrm{EtOH}}: 70 \mathrm{v} / \mathrm{v} \% ; Q_{\mathrm{CO}_{2}}\right.$ : $60 \mathrm{~mL} / \mathrm{min} ; V_{\mathrm{L}}: 25 \mathrm{~mL} ; T_{\text {absorption }}: 313.15 \mathrm{~K} ; T_{\text {desorption }}: 393.15 \mathrm{~K}$; $t_{\text {desorption }}: 30 \mathrm{~min}$; $P_{\text {regeneration: }}: 101.325 \mathrm{kPa}$ ) (color online)

的强信号峰, 在121.46、135.36 ppm处出现 $[\mathrm{Im}]^{-}$中的 C3、C4的共振峰，而17.03和56.90 ppm处分别对应乙 醇上的 $\mathrm{C} 1$ 和 $\mathrm{C} 2$ 两个碳原子的共振峰. 吸收 $\mathrm{CO}_{2}$ 后, 溶 液由均相转为液液两相. 上层溶液中仅有乙醇的共振 峰和较为微弱的 $[\mathrm{Im}]^{-}$共振峰, 未出现任何与 $\mathrm{CO}_{2}$ 产物 相关的共振峰, 说明上层贫相溶液的主要组分是乙醇. 而下层溶液在 163.23 和 $163.63 \mathrm{ppm}$ 处的共振峰分别对 应伯氨和仲氨上的羧基碳 $(\mathrm{C}=\mathrm{O})$, 而 $159.86 \mathrm{ppm}$ 处的共 振峰为 $\mathrm{HCO}_{3}{ }^{-} / \mathrm{CO}_{3}{ }^{2-}$. 此外, 下层溶液在 $158.75 \mathrm{ppm}$ 出 现明显的共振峰, 归属为碳酸乙酯 $\left(\mathrm{CH}_{3} \mathrm{CH}_{2} \mathrm{OCOO}^{-}\right)$, 这与Barbarossa等 ${ }^{[33]}$ 的研究发现乙醇可与 $\mathrm{CO}_{2}$ 反应会 形成碳酸乙酯相符.

该相变体系的解吸过程 ${ }^{13} \mathrm{C}$ NMR图谱结果如图 $3 b, c$ 所示. 下层富相溶液直接再生(图3b), 溶液中的氨 基甲酸酯 $\mathrm{C}=\mathrm{O}$ 的 $\mathrm{C}$ 原子共振峰的强度均随解吸过程不 断减弱, 即此阶段溶液的氨基甲酸酯浓度减少, 而碳酸 乙酯和 $\mathrm{HCO}_{3}{ }^{-} / \mathrm{CO}_{3}{ }^{2-}$ 也逐渐减少直至消失. 最终仍存 在较强的氨基甲酸酯信号峰, 说明产物无法彻底解吸, 与上节直接再生效率低的结果相符. 但当加入 $2 \mathrm{~mL}$ 贫 相溶液后(图3c), 产物峰(氨基甲酸酯、碳酸乙酯、 $\mathrm{HCO}_{3}{ }^{-} / \mathrm{CO}_{3}{ }^{2-}$ )强度骤减. 相较于直接再生, 后者最终 的氨基甲酸酯信号峰更弱, 进一步证实了加入适量的 贫相溶液可促进氨基甲酸酯的分解，进而提高再生 效率. 

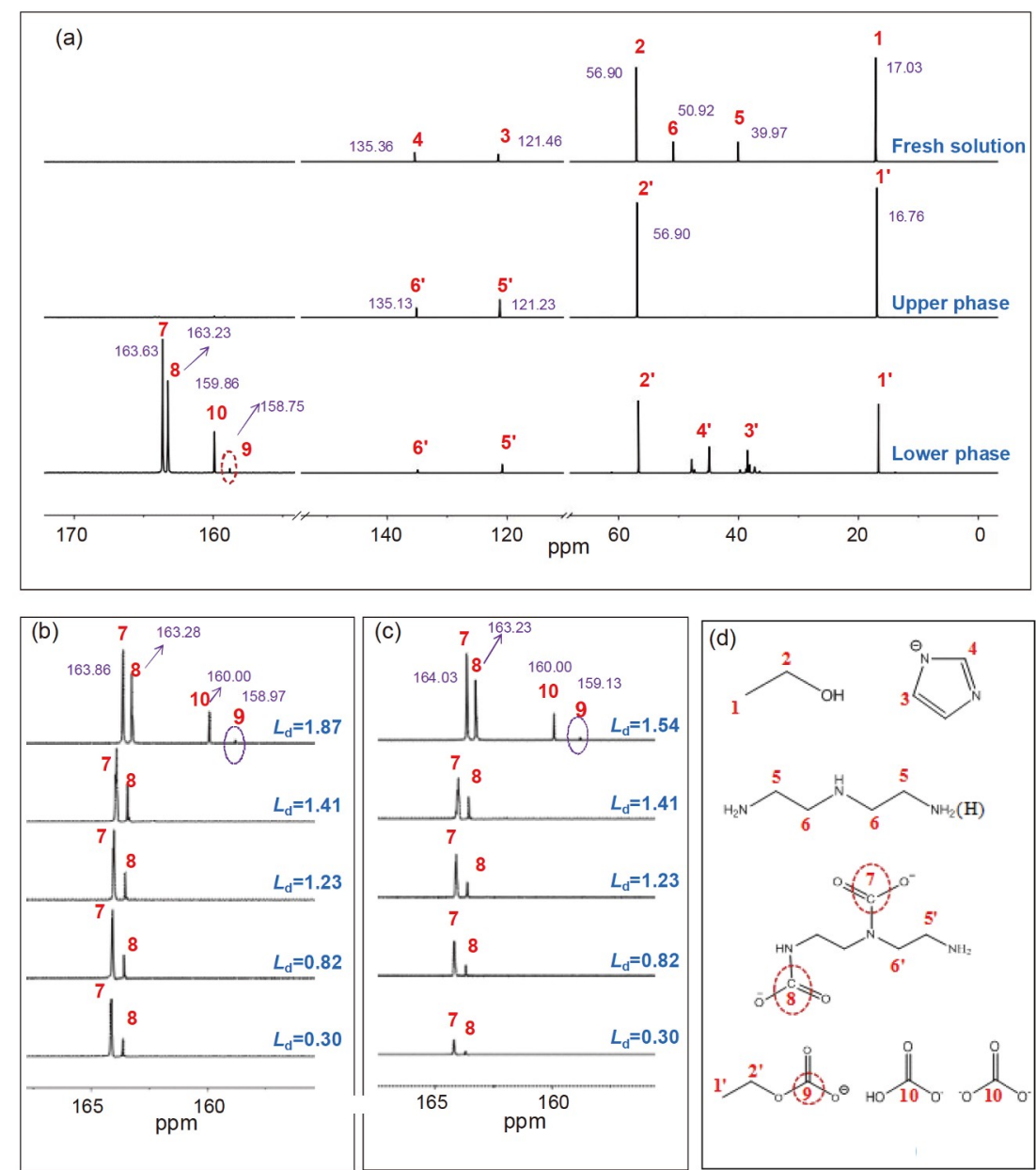

图 3 [DETAH] [Im]-乙醇- $\mathrm{H}_{2} \mathrm{O}$ 相变吸收剂捕集 $\mathrm{CO}_{2}$ 过程的核磁图谱. (a) 吸收剂吸收前后; (b) 不加贫相溶液再生; (c) 加 $4 \mathrm{~mL}$ 贫 相溶液再生 $\left(L_{\mathrm{d}}=\right.$ 解吸容量 $) ;(\mathrm{d})$ 分子结构和碳原子类型(网络版彩图)

Figure $3{ }^{13} \mathrm{C}$ NMR spectra of [DETAH] [Im]-EtOH- $\mathrm{H}_{2} \mathrm{O}$ biphasic solution during $\mathrm{CO}_{2}$ capture. (a) Absorbent solution before and after absorption; (b) regeneration without $\mathrm{CO}_{2}$-lean phase; (c) regeneration with $4 \mathrm{~mL}$ of the $\mathrm{CO}_{2}$-lean phase $\left(L_{\mathrm{d}}=\right.$ desorption loading); (d) molecular structure and types of carbon atoms (color online).

\subsection{2 量子化学计算}

基于上述 ${ }^{13} \mathrm{C}$ NMR结果表明, [DETAH][Im]-乙醇$\mathrm{H}_{2} \mathrm{O}$ 吸收体系吸收饱和后发生液-液相变，其中贫相溶 液以乙醇为主，而 $\mathrm{CO}_{2}$ 产物([Im]-H、DETA氨基甲酸 酯 $\left.\left(\mathrm{RN}^{+} \mathrm{COO}^{-}\right) 、 \mathrm{HCO}_{3}{ }^{-} 、 \mathrm{CO}_{3}{ }^{2-}\right)$ 主要富集到下层水溶 液中. 为了进一步探究相变及反应本质, 借助量子化学 计算进行考察.

通过DFT方法并选用B3LYP/6-31G**基组计算反 应产物与溶剂之间的相互作用情况, 结果列于表2. 从 表 2 可知, 反应产物与溶剂之间的氢键长度均小于 $2.7 \AA$, 且氢键角均大于 $120^{\circ}$, 表明产物与溶剂之间均可形成
氢键. 吸收剂与 $\mathrm{CO}_{2}$ 反应的产物 $\left(\mathrm{RN}^{+} \mathrm{COO}^{-} 、[\mathrm{Im}]-\mathrm{H}\right.$ 、 $\left.\mathrm{CO}_{3}{ }^{2-}, \mathrm{HCO}_{3}{ }^{-}\right)$与水之间形成的氢键键长分别为 $1.658 、 1.738 、 1.528$ 以及 $1.708 \AA$, 而产物与乙醇形成 的氢键长分别为 $1.699 、 1.645 、 1.556$ 以及 $1.725 \AA$. 产 物与水之间形成的氢键长度相较于与乙醇生成的氢键 短. 而氢键长度越短, 产物与溶剂之间的相互作用越 强. 因此, 产物更易与水结合形成氢键. 此外, 产物在 水中的极性大于在乙醇溶液中的极性, 导致产物向水 中聚集.

随后通过分子静电势图(MEPs)分析[DETAH][Im] 的阴、阳离子在 $70 v / v \%$ 乙醇体积分数的溶液环境中 分别与 $\mathrm{CO}_{2}$ 的反应位点. MEPs图中, 红色代表电荷密 
表 2 DFT/B3LYP/6-31++G(d,p) 基组下对 $\mathrm{CO}_{2}$ 产物与不同溶剂间氢键距离 $(\AA)$ 、键角 $\left({ }^{\circ}\right)$ 以及极性计算 ${ }^{a}$

Table 2 Calculation of hydrogen bond distance $(\AA)$, bond angle $\left({ }^{\circ}\right)$ and polarity between $\mathrm{CO}_{2}$ products and different solvents under DFT/B3LYP/6$31++\mathrm{G}(\mathrm{d}, \mathrm{P})$ level $^{\text {a) }}$

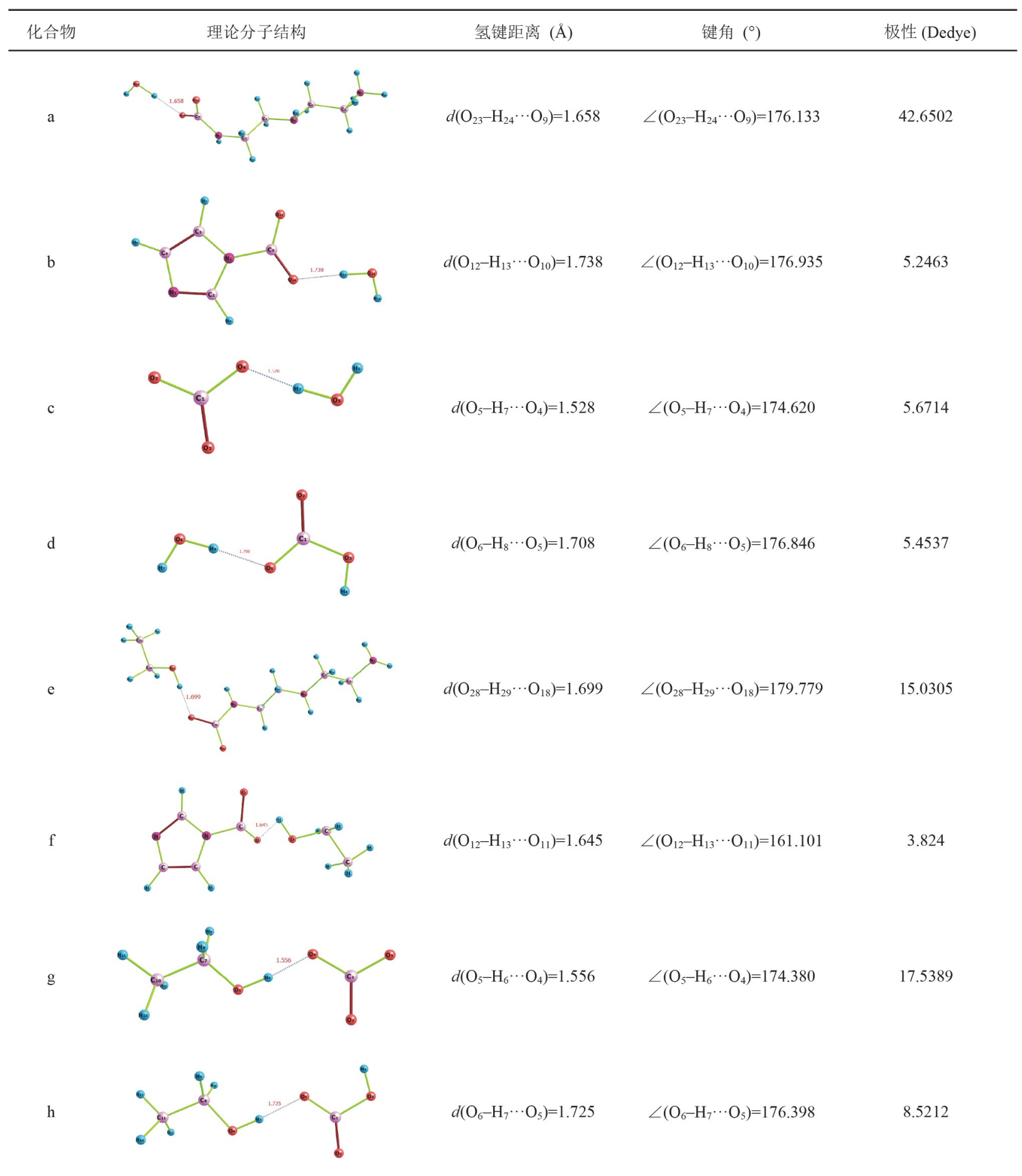

a) a、b、c和d分别代表 $\mathrm{RN}^{+} \mathrm{COO}^{-} 、[\mathrm{Im}]-\mathrm{H} 、 \mathrm{CO}_{3}{ }^{2-} 、 \mathrm{HCO}_{3}{ }^{-}$与水相互作用情况; e、f、g和h分别代表 $\mathrm{RN}^{+} \mathrm{COO}^{-} 、[\mathrm{Im}]-\mathrm{H} 、 \mathrm{CO}_{3}{ }^{2-} 、$ $\mathrm{HCO}_{3}{ }^{-}$与乙醇相互作用情况 
度低的局部原子区域，蓝色指示电荷密度高的局部原 子区域，黄色代表电中性区域. 如图4所示，[DETAH] $[\mathrm{Im}]$ 分子上的 $\mathrm{N}$ 原子区域的电荷密度高，而 $\mathrm{C}$ 原子、 $\mathrm{H}$ 原子区域处的电荷密度相对较低，这说明 $\mathrm{N}$ 原子有着 更高的电负性，吸收过程中可与电负性较弱的 $\mathrm{CO}_{2}$ 中 的 $\mathrm{C}$ 原子相结合, 从而提高吸收性能.

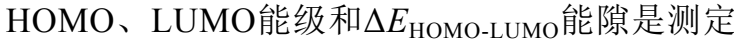
化合物间的化学稳定性的重要参数. 能隙较大则分子
间表现出良好的化学稳定性，能隙较小则分子间具有 较高的化学反应性 ${ }^{[34]}$. 其中, 产物间的能隙采用DFT/ B3LYP/6-31G $(d, p)$ 法计算可得. 由表3可知, 阳离子氨 基与 $\mathrm{CO}_{2}$ 的反应产物氨基甲酸酯 $\left(\mathrm{RN}^{+} \mathrm{COO}^{-}\right)$与唑基阴 离子质子化的 $[\mathrm{Im}]-\mathrm{H}$ 结合的能隙为 $0.2068 \mathrm{eV}$, 低于 $\mathrm{RN}^{+} \mathrm{COO}^{-}$和 $\mathrm{RN}^{+} \mathrm{COO}^{-}$的能隙 $(0.233 \mathrm{eV})$ 和 $\mathrm{RN}^{+} \mathrm{COO}^{-}$[Im]- $\mathrm{COO}^{-}$的能隙 $(0.2145 \mathrm{eV})$, 说明 $[\mathrm{Im}]-\mathrm{H}$ 会与 $\mathrm{RN}^{+} \mathrm{COO}^{-}$反应促进 $\mathrm{HCO}_{3}{ }^{-} / \mathrm{CO}_{3}{ }^{2-}$ 的形成, 因此,

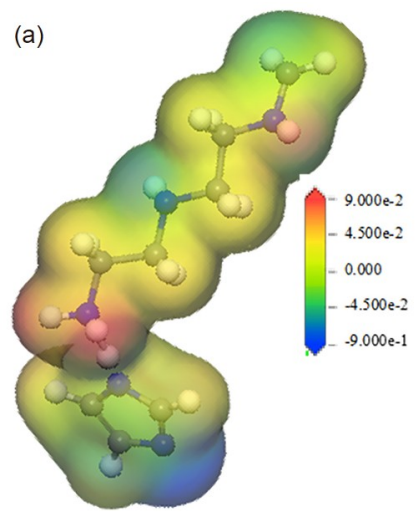

(b)

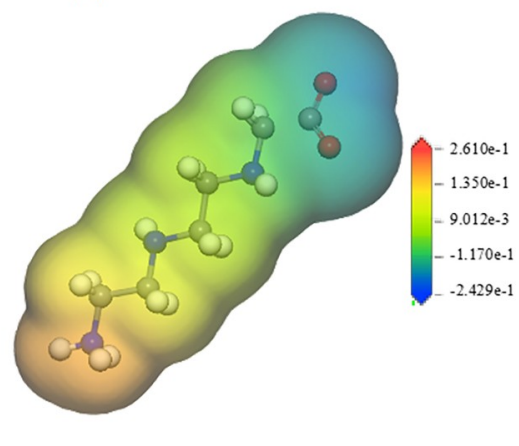

(c)

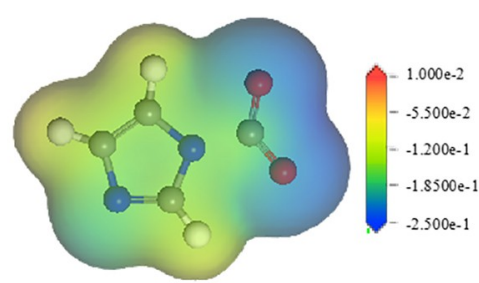

图 4 [DETAH] $[\mathrm{Im}](\mathrm{a}) 、[\mathrm{DETAH}]^{+}-\mathrm{CO}_{2}(\mathrm{~b}) 、[\mathrm{Im}]^{-}-\mathrm{CO}_{2}(\mathrm{c})$ 的分子静电势图 $(\mathrm{MEPs})$ (红色=正、蓝色=负、黄色=零电势) (网 络版彩图)

Figure 4 The molecular electrostatic potential (MEPs) for $[\mathrm{DETAH}][\mathrm{Im}](\mathrm{a}) 、[\mathrm{DETAH}]^{+}-\mathrm{CO}_{2}(\mathrm{~b}) 、[\mathrm{Im}]^{-}-\mathrm{CO}_{2}(\mathrm{c})($ red=positive, blue=negative and yellow=zero potentials) (color online).

表 3 计算 $\mathrm{RN}^{+} \mathrm{COO}^{-}-\mathrm{RN}^{+} \mathrm{COO}^{-}$(a)、 $\mathrm{RN}^{+} \mathrm{COO}^{-}-[\mathrm{Im}]-\mathrm{H}$ (b)和 $\mathrm{RN}^{+} \mathrm{COO}^{-}$-[Im]-COO${ }^{-}$(c)物质在 $70 \%$ 乙醇 $/ 30 \%$ 水混合溶液中的 最优构型、HOMO和LUMO

Table 3 The calculated optimized structures, $\mathrm{HOMO}$ and LUMO orbitals of the molecular structure $\mathrm{RN}^{+} \mathrm{COO}^{-}-\mathrm{RN}^{+} \mathrm{COO}^{-}$(a), $\mathrm{RN}^{+} \mathrm{COO}^{-}-[\mathrm{Im}]-\mathrm{H}^{(b)}$ and $\mathrm{RN}^{+} \mathrm{COO}^{-}-[\mathrm{Im}]-\mathrm{COO}^{-}$(c) in $70 \% \mathrm{EtOH} / 30 \% \mathrm{H}_{2} \mathrm{O}$ solution

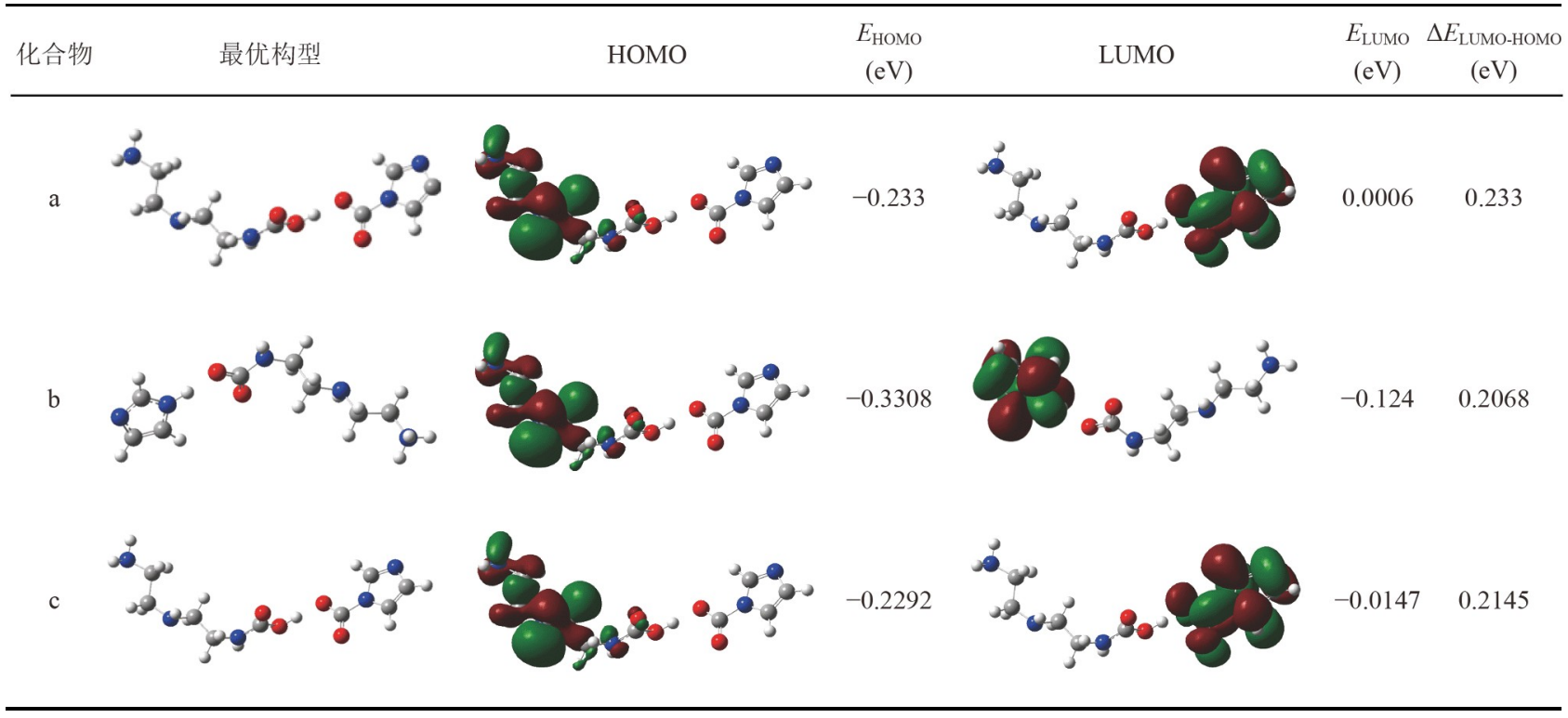


$\mathrm{RN}^{+} \mathrm{COO}^{-}$和 $[\mathrm{Im}]-\mathrm{H}$ 之间活跃的相互作用更有利于饱 和富相溶液中解吸出 $\mathrm{CO}_{2}$. 因此, 吸收剂中唑基阴离子 质子化的 $[\mathrm{Im}]-\mathrm{H}$ 会促进反应产物氨基甲酸酯 $\mathrm{RN}^{+} \mathrm{COO}^{-}$ 水解成更易再生的 $\mathrm{HCO}_{3}{ }^{-} / \mathrm{CO}_{3}{ }^{2-}$, 从而促进再生性能 的提高.

\subsection{3 相变吸收剂捕集 $\mathrm{CO}_{2}$ 反应及相变机理}

通过以上实验及量子化学计算计算, 阐明了 [DETAH] [Im]-乙醇- $\mathrm{H}_{2} \mathrm{O}$ 捕集 $\mathrm{CO}_{2}$ 的吸收-解吸机理和 相变机制. 在吸收过程中, $[\mathrm{DETAH}][\mathrm{Im}]$ 中的阳离子 $\left([\mathrm{DETAH}]^{+}, \mathrm{RNH}^{+}\right)$和阴离子 $\left([\mathrm{Im}]^{-}\right)$均可与 $\mathrm{CO}_{2}$ 发生化 学反应. 其中阳离子 $\left(\mathrm{RNH}^{+}\right)$含有伯胺基团和仲胺基团, $\mathrm{RNH}^{+}$中的胺基与 $\mathrm{CO}_{2}$ 反应生成两性离子，并继续与 $\mathrm{RNH}^{+}$反应生成氨基甲酸酯 $\left(\mathrm{RN}^{+} \mathrm{COO}^{-}\right)^{[35]}$. 反应式 如下:

$$
\mathrm{CO}_{2}+\mathrm{RNH}^{+} \rightleftharpoons \mathrm{RNH}^{2+} \mathrm{COO}^{-}
$$$$
\mathrm{RNH}^{2+} \mathrm{COO}^{-}+\mathrm{RNH}^{+} \rightleftharpoons \mathrm{RN}^{+} \mathrm{COO}^{-}+\mathrm{RNH}_{2}{ }^{2+}
$$

结合方程式(3)、(4)可推导出 $\mathrm{CO}_{2}+2 \mathrm{RNH}^{+} \rightleftharpoons \mathrm{RN}^{+} \mathrm{COO}^{-}+\mathrm{RNH}_{2}{ }^{2+}$

通常, $1 \mathrm{~mol} \mathrm{CO}$ 会消耗 $2 \mathrm{~mol}$ 伯胺或仲胺, 因而胺 类吸收剂极大受限于其吸收容量. 而 $\mathrm{Wu}$ 等 ${ }^{[25]}$ 研究发现 唑基功能化离子液体在水相中可与 $\mathrm{CO}_{2}$ 发生等摩尔反 应, 并生成咪唑-氨基甲酸酯([Im]-COO${ }^{-}$), 可提高吸收 剂的 $\mathrm{CO}_{2}$ 负载量. 因此, $[\mathrm{DETAH}][\mathrm{Im}]$ 的阴离子 $\left([\mathrm{Im}]^{-}\right)$ 与 $\mathrm{CO}_{2}$ 反应如下:

$[\mathrm{Im}]^{-}+\mathrm{CO}_{2} \rightleftharpoons[\mathrm{Im}]-\mathrm{COO}^{-}$

随着反应的进行, [DETAH] [Im] 被消耗, 而溶液中 的 $\mathrm{CO}_{2}$ 负载量增加. 随后吸收产物及 $\mathrm{CO}_{2}$ 均会发生水解 反应, 生成 $\mathrm{HCO}_{3}{ }^{-} / \mathrm{CO}_{3}{ }^{2-}$, 具体反应如下:

$$
\begin{aligned}
& \mathrm{RN}^{+} \mathrm{COO}^{-}+\mathrm{H}_{2} \mathrm{O} \rightleftharpoons \mathrm{RNH}_{2}{ }^{2+}+\mathrm{CO}_{3}^{2-} \\
& {[\mathrm{Im}]-\mathrm{COO}^{-}+\mathrm{H}_{2} \mathrm{O} \rightleftharpoons[\mathrm{Im}]-\mathrm{H}+\mathrm{HCO}_{3}^{-}} \\
& \mathrm{CO}_{2}+\mathrm{H}_{2} \mathrm{O} \rightleftharpoons \mathrm{H}^{+}+\mathrm{HCO}_{3}^{-}
\end{aligned}
$$

同时，唑基阴离子质子化后形成的 $[\mathrm{Im}]-\mathrm{H}$ 和乙醇 均可促进 $\mathrm{RN}^{+} \mathrm{COO}^{-}$水解反应, 这有利于促进 $\mathrm{RN}^{+} \mathrm{COO}^{-}$ 的分解和氨基 $\mathrm{RNH}^{+}$的回收, 保证了 [DETAH] [Im] 溶液 的高吸收容量和解吸效率.

$$
\begin{aligned}
& \mathrm{RN}^{+} \mathrm{COO}^{-}+[\mathrm{Im}]-\mathrm{H}+\mathrm{OH}^{-} \rightleftharpoons \mathrm{RNH}^{+}+[\mathrm{Im}]^{-}+\mathrm{HCO}_{3}{ }^{-} \\
& \mathrm{RN}^{+} \mathrm{COO}^{-}+\mathrm{CH}_{3} \mathrm{CH}_{2} \mathrm{OH} \rightarrow \mathrm{RNH}^{+}+\mathrm{CH}_{3} \mathrm{CH}_{2} \mathrm{OCOO}^{-}
\end{aligned}
$$

[DETAH] [Im]-乙醇- $\mathrm{H}_{2} \mathrm{O}$ 相变吸收剂吸收饱和后,
吸收产物主要为 $[\mathrm{DETAH}]^{+}$衍生的氨基甲酸酯 $\left(\mathrm{RN}^{+}\right.$ $\left.\mathrm{COO}^{-}\right) 、[\mathrm{Im}]-\mathrm{H}$ 和 $\mathrm{HCO}_{3}{ }^{-} / \mathrm{CO}_{3}{ }^{2-}$ 、碳酸乙酯 $\left(\mathrm{CH}_{3} \mathrm{CH}_{2}{ }^{-}\right.$ $\mathrm{OCOO}^{-}$). 由于这些产物在水溶液中的极性强于在乙 醇溶液中, 且乙醇密度比水小, 因此乙醇逐渐向上层 移动而产物向下层移动富集于溶液水相, 最终形成上 层乙醇贫相和下层水溶液富相的液液两相. 乙醇在吸 收过程主要起到分相的作用, 以及少量参与 $\mathrm{CO}_{2}$ 吸收 反应生成 $\mathrm{CH}_{3} \mathrm{CH}_{2} \mathrm{OCOO}^{-}$.

根据 ${ }^{13} \mathrm{C}$ NMR图谱可知, 所有产物通过热解反应 得以再生并重新生成原物质, 解吸与吸收互为逆过程. 因此, 有一部分 $\mathrm{HCO}_{3}{ }^{-} / \mathrm{CO}_{3}{ }^{2-}$ 在热解过程中释放出 $\mathrm{CO}_{2}$, 另一部分则与 $\mathrm{RNH}_{2}^{+}$反应生成 $\mathrm{RN}^{+} \mathrm{COO}^{-}$. 富相溶 液的再生性取决于产物中最难分解的 $\mathrm{RN}^{+} \mathrm{COO}^{-}$的再 生. 离子液体中质子化的咪唑 $([\mathrm{Im}]-\mathrm{H})$ 后会与 $\mathrm{RN}^{+} \mathrm{COO}^{-}$发生反应, 消耗 $\mathrm{RN}^{+} \mathrm{COO}^{-}$并促进其再生. 此 外, $\mathrm{RN}^{+} \mathrm{COO}^{-}$还会与加入的 $2 \mathrm{~mL}$ 贫相乙醇反应生成更 易热解吸的碳酸乙酯, 使其被进一步消耗, 提高再生效 率. 该结论合理解释了解吸液中添加贫相溶液可提高 再生效率的现象.

由于 $\mathrm{HCO}_{3}{ }^{-} / \mathrm{CO}_{3}{ }^{2-}$ 一部分在热解中释放了 $\mathrm{CO}_{2}$, 其 他部分则与 $\mathrm{RNH}_{2}{ }^{2+}$ 反应生成 $\mathrm{RN}^{+} \mathrm{COO}^{-}$, 所以 $\mathrm{HCO}_{3}{ }^{-}$/ $\mathrm{CO}_{3}{ }^{2-}$ 的信号峰强度持续下降. 具体反应式如下:

$\mathrm{CO}_{3}{ }^{2-}+2 \mathrm{H}^{+} \rightleftharpoons \mathrm{H}_{2} \mathrm{O}+\mathrm{CO}_{2}$

$\mathrm{HCO}_{3}{ }^{-}+\mathrm{H}^{+} \rightleftharpoons \mathrm{H}_{2} \mathrm{O}+\mathrm{CO}_{2}$

$2 \mathrm{HCO}_{3}{ }^{-}+\mathrm{RNH}_{2}{ }^{2+} \rightleftharpoons \mathrm{RN}^{+} \mathrm{COO}^{-}+2 \mathrm{H}_{2} \mathrm{O}+\mathrm{CO}_{2}$

$\mathrm{RN}^{+} \mathrm{COO}^{-}+\mathrm{H}^{+} \rightleftharpoons \mathrm{RNH}^{+}+\mathrm{CO}_{2}$

基于上述分析, [DETAH] [Im]-乙醇- $\mathrm{H}_{2} \mathrm{O}$ 相变吸收 剂捕集 $\mathrm{CO}_{2}$ 的反应及相变机制如图5所示. [DETAH] [Im] 作为主吸收剂吸收 $\mathrm{CO}_{2}$ 生成氨基甲酸酯和 $\mathrm{HCO}_{3}{ }^{-} / \mathrm{CO}_{3}{ }^{2-}$. 产物和溶剂的极性差异和氢键作用情况, 影响 $\mathrm{CO}_{2}$ 产 物在不同溶剂中的分配情况, 最终高密度的产物富集 在下层水中, 而乙醇聚集在溶液上层. 因此, 乙醇在吸 收剂中充当相分离促进剂的角色. 在解吸过程中, [DE$\mathrm{TAH}][\mathrm{Im}]$ 离子液体的唑基阴离子质子化后可消耗氨 基甲酸酯, 而乙醇也可以通过促进氨基甲酸酯转化为 碳酸乙酯, 共同提高饱和富相溶液的再生性能.

\section{4 结论}

本文构建了 [DETAH] [Im]-乙醇- $\mathrm{H}_{2} \mathrm{O}$ 三元相变吸 收剂捕集 $\mathrm{CO}_{2}$, 考察了其吸收-解吸性能, 阐明了其捕集 


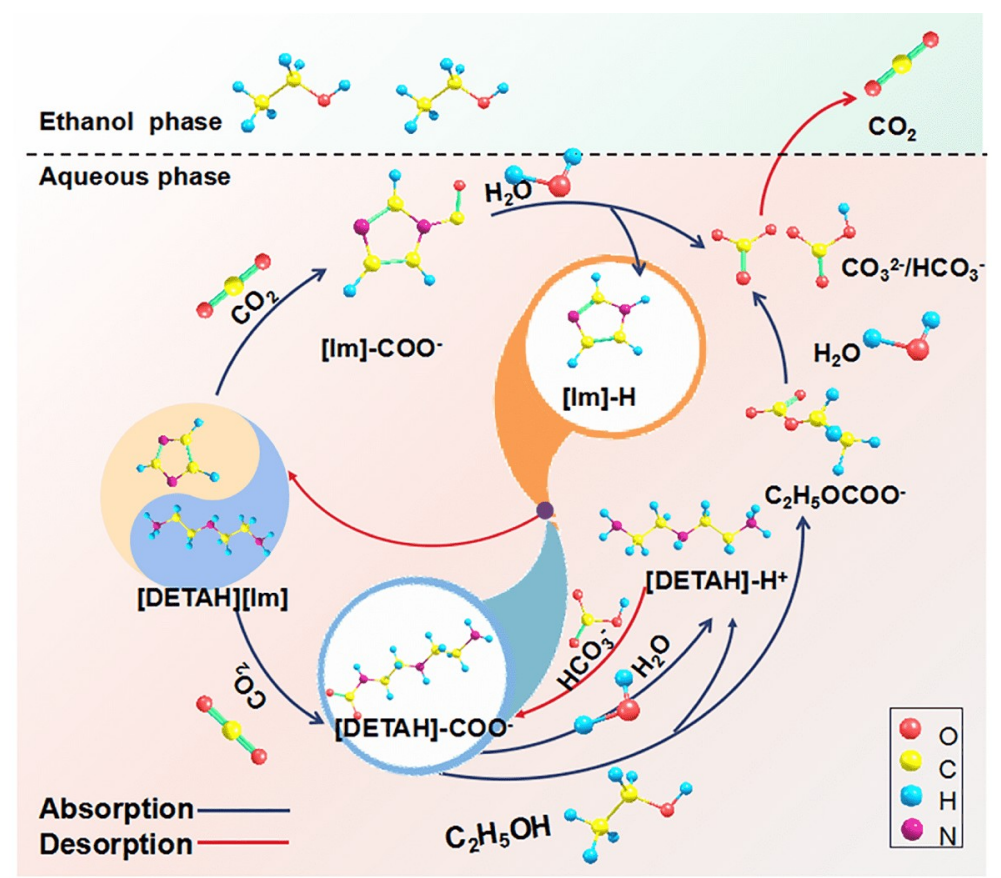

图 5 [DETAH] [Im]-乙醇- $\mathrm{H}_{2} \mathrm{O}$ 吸收剂捕集 $\mathrm{CO}_{2}$ 机理图(网络版彩图)

Figure 5 The mechanism of $\mathrm{CO}_{2}$ capture into [DETAH][Im]-EtOH- $\mathrm{H}_{2} \mathrm{O}$ (color online).

$\mathrm{CO}_{2}$ 的反应及相变机制. 所得结果如下：(1) 当[DE$\mathrm{TAH}][\mathrm{Im}]$ 浓度为 $1 \mathrm{kmol} / \mathrm{m}^{3}$ 、乙醇在醇-水混合溶剂中 配比为 $70 v / v \%$ 时, 该三元吸收剂为最优相变吸收剂体 系，吸收饱和后发生液液分相，其总吸收容量为 $1.548 \mathrm{~mol} \mathrm{CO} / \mathrm{mol}$ absorbent, 富相溶液 $\mathrm{CO}_{2}$ 吸收容量 占总容量的 $92 \%$ 而体积仅占总体积的 $32 \%$. (2) 吸收过 程中, $[\mathrm{DETAH}][\mathrm{Im}]$ 作为主吸收剂, 阳离子与 $\mathrm{CO}_{2}$ 反应 生成氨基甲酸酯 $\left(\mathrm{RN}^{+} \mathrm{COO}^{-}\right)$, 而唑基阴离子与 $\mathrm{CO}_{2}$ 发 生等摩尔反应生成 $\mathrm{HCO}_{3}{ }^{-} / \mathrm{CO}_{3}{ }^{2-}$, 保证了体系高效的
$\mathrm{CO}_{2}$ 捕集能力. 同时, [ $\left.\mathrm{Im}\right]-\mathrm{H}$ 和乙醇可促进氨基甲酸酯 $\left(\mathrm{RN}^{+} \mathrm{COO}^{-}\right)$的分解, 并生成 $\mathrm{HCO}_{3}{ }^{-} / \mathrm{CO}_{3}{ }^{2-}$ 和碳酸乙酯 产物, 而溶液中也伴随发生 $\mathrm{CO}_{2}$ 的水解反应. 解吸反应 是吸收反应的逆过程, [Im]-H和乙醇的作用共同保证 了再生液的高再生效率. (3) $\mathrm{CO}_{2}$ 吸收产物与水之间的 强氢键作用力和高极性会导致溶液体系局域密度和性 质的差异, 最终导致水相和乙醇相的形成, $\mathrm{CO}_{2}$ 产物富 集在下层的水相中, 上层的乙醇相中仅含有少量的 $\mathrm{CO}_{2}$ 吸收物种.

\section{补充材料}

本文的补充材料见网络版http://chemcn.scichina.com. 补充材料为作者提供的原始数据, 作者对其学术质量和内容负责.

\section{参考文献}

1 Yu BY, Zhao GP, An RY, Chen JM, Tan JX, Li XY. J B Inst Technol, 2021, 23: 17-24 (in Chinese) [余碧芗, 赵光普, 安润颖, 陈景明, 谭锦潇, 李 晓易. 北京理工大学学报, 2021, 23: 17-24]

2 Jiang KJ. China Sci Daily, 2021. (in Chinese) [姜克隽. 中国科学报, 2021. doi: 10.28514/n.cnki.nkxsb.2021.000068]

3 Bui M, Adjiman CS, Bardow A, Anthony EJ, Boston A, Brown S, Fennell PS, Fuss S, Galindo A, Hackett LA, Hallett JP, Herzog HJ, Jackson G, Kemper J, Krevor S, Maitland GC, Matuszewski M, Metcalfe IS, Petit C, Puxty G, Reimer J, Reiner DM, Rubin ES, Scott SA, Shah N, Smit B, Trusler JPM, Webley P, Wilcox J, Mac Dowell N. Energy Environ Sci, 2018, 11: 1062-1176

4 Liang ZH, Rongwong W, Liu H, Fu K, Gao H, Cao F, Zhang R, Sema T, Henni A, Sumon K, Nath D, Gelowitz D, Srisang W, Saiwan C, 
Benamor A, Al-Marri M, Shi H, Supap T, Chan C, Zhou Q, Abu-Zahra M, Wilson M, Olson W, Idem R, Tontiwachwuthikul PP. Int J Greenh Gas Control, 2015, 40: 26-54

5 Zhang S, Du M, Shao P, Wang L, Ye J, Chen J, Chen J. Environ Sci Technol, 2018, 52: 12708-12716

6 Zhang X, Zhang X, Liu H, Li W, Xiao M, Gao H, Liang Z. Appl Energy, 2017, 202: 673-684

7 Qian Y, Jing G, Lv B, Zhou Z. Energy Fuels, 2017, 31: 4202-4210

8 Cuccia L, Dugay J, Bontemps D, Louis-Louisy M, Morand T, Kanniche M, Bellosta V, Vial J. Int J Greenh Gas Control, 2019, 80: 43-53

9 Zhang S, Shen Y, Wang L, Chen J, Lu Y. Appl Energy, 2019, 239: 876-897

10 Wang L, Zhang Y, Wang R, Li Q, Zhang S, Li M, Liu J, Chen B. Environ Sci Technol, 2018, 52: 14556-14563

11 Tu WW, Fang JW, Li ZS, Mao ML, Jin XH, Liu XY, Zhang WD. Sci Sin Chim, 2018, 48: 641-647 (in Chinese) [涂巍巍, 方佳伟, 李竹石, 毛梦 琳, 金显杭, 刘晓阳, 张卫东. 中国科学: 化学, 2018, 48: 641-647]

12 Zhang BX, Li HT, Chen X, Mao SB, Guo BS, Ji Y. Energ Chem Ind, 2020, 41: 8-11 (in Chinese) [张宝幸, 李海涛, 陈曦, 毛松柏, 郭本帅, 季燕. 能源化工, 2020, 41: 8-11]

13 Wang R, Liu S, Wang L, Li Q, Zhang S, Chen B, Jiang L, Zhang Y. Appl Energy, 2019, 242: 302-310

14 Zhu K, Lu H, Liu C, Wu K, Jiang W, Cheng J, Tang S, Yue H, Liu Y, Liang B. Ind Eng Chem Res, 2019, 58: 3811-3821

15 Shen S, Bian Y, Zhao Y. Int J Greenh Gas Control, 2017, 56: 1-11

16 Luo W, Guo D, Zheng J, Gao S, Chen J. Int J Greenh Gas Control, 2016, 53: 141-148

17 Zhang S, Sun J, Zhang X, Xin J, Miao Q, Wang J. Chem Soc Rev, 2014, 43: 7838-7869

18 Sun C, Liu F, Shen L, Li SJ, Li W. J Chem Eng Chin Univ, 2020, 34: 1135-1142 (in Chinese) [孙铖, 刘凡, 沈丽, 李素静, 李伟. 高校化学工程学 报. 2020, 34: 1135-1142]

19 Yuan G, Zhao Y, Wu Y, Li R, Chen Y, Xu D, Liu Z. Sci China Chem, 2017, 60: 958-963

20 Zhou HC. Application of ionic liquid phase change system in $\mathrm{CO}_{2}$ capture. Dissertation for Master's Degree. Beijing: Beijing University of Chemical Technology, 2020 [周海铖. 离子液体相变体系在 $\mathrm{CO}_{2}$ 捕集中的应用研究. 硕士学位论文. 北京: 北京化工大学, 2020]

21 Seo S, Simoni LD, Ma M, DeSilva MA, Huang Y, Stadtherr MA, Brennecke JF. Energy Fuels, 2014, 28: 5968-5977

22 Xia PW, Ding BH, Zhang PJ, Zang SL, Deng GC, Wang Q. Appl Chem Ind, 2019, 48: 1469-1473 (in Chinese) [夏裴文, 丁保宏, 张鹏军, 蔵树 良, 邓桂春, 王强. 应用化工, 2019, 48: 1469-1473]

23 Kassim MA, Sairi NA, Yusoff R, Alias Y, Aroua MK. Ind Eng Chem Res, 2016, 55: 7992-8001

24 Zhou H, Xu X, Chen X, Yu G. Int J Greenh Gas Control, 2020, 98: 103068

25 Wu J, Lv B, Wu X, Zhou Z, Jing G. ACS Sustain Chem Eng, 2019, 7: 7312-7323

26 Jing G, Qian Y, Zhou X, Lv B, Zhou Z. ACS Sustain Chem Eng, 2018, 6: 1182-1191

27 Xie HB, Zhou Y, Zhang Y, Johnson JK. J Phys Chem A, 2010, 114: 11844-11852

28 Hwang GS, Stowe HM, Paek E, Manogaran D. Phys Chem Chem Phys, 2015, 17: 831-839

29 Lv B, Wu K, Zhou Z, Jing G. Int J Greenh Gas Control, 2019, 91: 102846

30 Arivazhagan M, Subhasini VP. Spectrochim Acta Part A-Mol Biomol Spectr, 2012, 91: 402-410

31 Masoud MS, Ali AE, Shaker MA, Elasala GS. Spectrochim Acta Part A-Mol Biomol Spectr, 2012, 90: 93-108

32 Rahmouni NT, Bensiradj NH, Megatli SA, Djebbar S, Benali Baitich O. Spectrochim Acta Part A-Mol Biomol Spectr, 2019, 213: 235-248

Barbarossa V, Barzagli F, Mani F, Lai S, Stoppioni P, Vanga G. RSC Adv, 2013, 3: 12349-12355

Ermiş E, Durmuş K, Aygüzer ÖU, Berber H, Güllü M. J Mol Structure, 2018, 1168: 115-126

Zhou X, Jing G, Lv B, Liu F, Zhou Z. Appl Energy, 2019, 235: 379-390 


\title{
Mechanism of $\mathrm{CO}_{2}$ capture into amino-azolyl dual-functionalized ionic liquid biphasic solvent
}

\author{
Bihong Lv, Xiaohui Zhan, Xin Li, Zuoming Zhou, Guohua Jing* \\ College of Chemical Engineering, Huaqiao University, Xiamen 361021, China \\ *Corresponding author (email: zhoujing@hqu.edu.cn)
}

\begin{abstract}
Biphasic solvents exhibit great potential for saving energy in $\mathrm{CO}_{2}$ capture. However, the efficiency of the available amine-based biphasic solvent is compromised due to the high viscosity and low regeneration efficiency of the rich phase solution. In this work, a novel amino-azolyl dual-functional ionic liquid/ethanol/water ([DETAH][Im]/EtOH/ $\mathrm{H}_{2} \mathrm{O}$ ) biphasic solvent for $\mathrm{CO}_{2}$ capture was proposed via dissolution of a diethylenetriamine-imidazole functionalized ionic liquid ([DETAH][Im]) in the mixed ethanol-water solvent. This biphasic solvent transitioned from homogeneous phase to liquid-liquid phase after saturation of $\mathrm{CO}_{2}$ capture. And the formed products during $\mathrm{CO}_{2}$ capture were mainly concentrated in the lower phase. When the concentration of ethanol was $70 v / v \%$, the $\mathrm{CO}_{2}$ loading of the rich solution ( $1.548 \mathrm{~mol} \mathrm{CO} / \mathrm{mol}$ absorbent) accounted for $92 \%$ of the total $\mathrm{CO}_{2}$ loading whereas the volume only accounted for $32 \%$. And ethanol was used as a regeneration activator, which could guarantee that the rich liquid retained more than $90 \%$ of its initial load after four times of regeneration. The reaction of both the amino and azole group functional groups in [DETAH] [Im] with $\mathrm{CO}_{2}$ ensured the efficient $\mathrm{CO}_{2}$ loading of the biphasic solvent. In addition, [Im]-H and ethanol can enhance the hydrolysis of carbamate to form easily decomposed $\mathrm{HCO}_{3}{ }^{-} / \mathrm{CO}_{3}{ }^{2-}$ and ethyl carbonate and the high regeneration efficiency of the rich liquid can be obtained. The hydrogen bond and polarity products with high density in water were stronger than ethanol, leading to the enrichment of the products in the lower aqueous solution. Nevertheless, ethanol with low density is in the upper layer, eventually forming liquid-liquid phase transition.
\end{abstract}

Keywords: carbon dioxide, functionalized ionic liquids, biphasic solvent, mechanism

doi: $10.1360 /$ SSC-2021-0026 